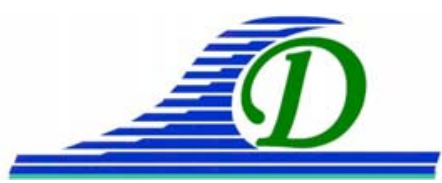

XIII ${ }^{\text {èmes }}$ Journées Nationales Génie Côtier - Génie Civil

Dunkerque, 2-4 juillet 2014

DOI:10.5150/jngcgc.2014.041 ～(C) Editions Paralia CFL

disponible en ligne - http://www.paralia.fr - available online

\title{
Analyse morpho-sédimentaire des cours d'eau du barrage de Bouhanifia
}

\author{
Habib GUESSAS ${ }^{1}$, Belkacem MEKERTA ${ }^{1}$, Abdélaziz SEMCHA ${ }^{1}$, \\ Fatiha KAZI-AOUEL ${ }^{1}$, Mohammed AMEUR ${ }^{1}$, Jean-Pierre TROALEN ${ }^{2}$
}

1. Ecole Nationale Polytechnique d'Oran, BP 1523 Oran El M’Naouer, Algérie. habibmag@yahoo.fr,mekertab@yahoo.fr

2. Université de Reims Champagne Ardenne, Laboratoire Génie Civil, Moulin de la Housse, BP 1039, 51687 Reims cedex 2, France.

mjp.troalen@orange.fr

\section{Résumé :}

Les conséquences de l'envasement des barrages qui se traduisent par la perte des volumes utiles des retenues, continuent à poser des problèmes aux gestionnaires de l’Agence Nationale des Barrages et Transfert en Algérie. La nature des précipitations dans nos régions à climat semi-aride, accentue la dégradation des berges des bassins versants.

Le barrage de Bouhanifia qui fait l'objet de notre étude, vue sa position géographique dans la région de Mascara (ouest algérien), se situe sur l'axe de l'oued El Hammam en aval du barrage de Ouizert et en amont de celui de Fergoug. Des opérations de dragage estimées à 6 millions de $\mathrm{m}^{3}$ ont débuté en juin 2013, ce qui constitue un terrain expérimental concret pour la recherche de solutions sur les moyens curatives et préventives de lutte à mettre en œuvre contre l'accumulation des sédiments dans la retenue.

Les paramètres qui régissent et caractérisent les bassins versants, la morphologie des cours d'eau et le transport solide, doivent être étudiés pour permettre des applications en relation avec les conditions actuelles de l’ouvrage. Nous avons déterminé les propriétés essentielles des cours d'eau, à savoir : la pente, la sinuosité et la rugosité. Les différents résultats permettent de connaître les cours d'eau qui nécessitent des interventions de maintenance et d'entretien, pour minimiser l'érosion et par conséquent contribuer à l'entretien du barrage de Bouhanifia en aval contre l'envasement.

Mots-clés : Barrage de Bouhanifia, Précipitations, Envasement, Morphologie des cours d’eau, Pente, Sinuosité, Rugosité, Maintenance préventive.

\section{Introduction}

Parmi tant d'approches et de techniques sont mises en œuvre en Algérie pour la conservation et la préservation des ressources hydriques, la maintenance des ouvrages hydrauliques vient apporter sa contribution pour lutter contre les pertes importantes de volumes d'eau occasionnés par les matériaux qui sont piégés chaque année dans les 


\section{Thème 2 - Dynamique sédimentaire}

retenues de barrages. Le comblement est estimé en moyenne à $25 \%$ par l'Agence Nationale des Barrages et Transfert en Algérie (GOMER, 1994) et à 20 millions de $\mathrm{m}^{3}$ par an (REMINI \& HALLOUCHE, 2005). Cette particularité contribue à réduire de $0,3 \%$ par an les capacités de stockage de l'eau, avec potentiel mobilisé de 6,2 milliards de $\mathrm{m}^{3}$.

La lutte contre l'envasement de ces ouvrages est devenue une nécessité voire un défi pour sauvegarder l'eau indispensable à notre vie et celle des générations futures.

Notre travail vise à mettre en relief l'état de l'envasement du barrage de Bouhanifia, et de proposer des techniques innovantes en matière de lutte préventive pour endiguer ce phénomène.

\section{La zone d'étude}

Le barrage de Bouhanifia qui fait l'objet de notre étude est construit sur l'oued El Hammam dans le bassin versant de la Macta, situé au nord-ouest de l'Algérie (voir figure 1), (BOUANANI, 1999).

Le bassin versant de la Macta d'une superficie de $14390 \mathrm{~km}^{2}$, est drainé par deux principaux cours d'eau, l'oued Mebtouh (138 km de longueur) à l'ouest et l'oued El Hammam à l'est (150 km de longueur; y compris son affluent principal l'oued Taria et son prolongement l'oued Aine Fekane).

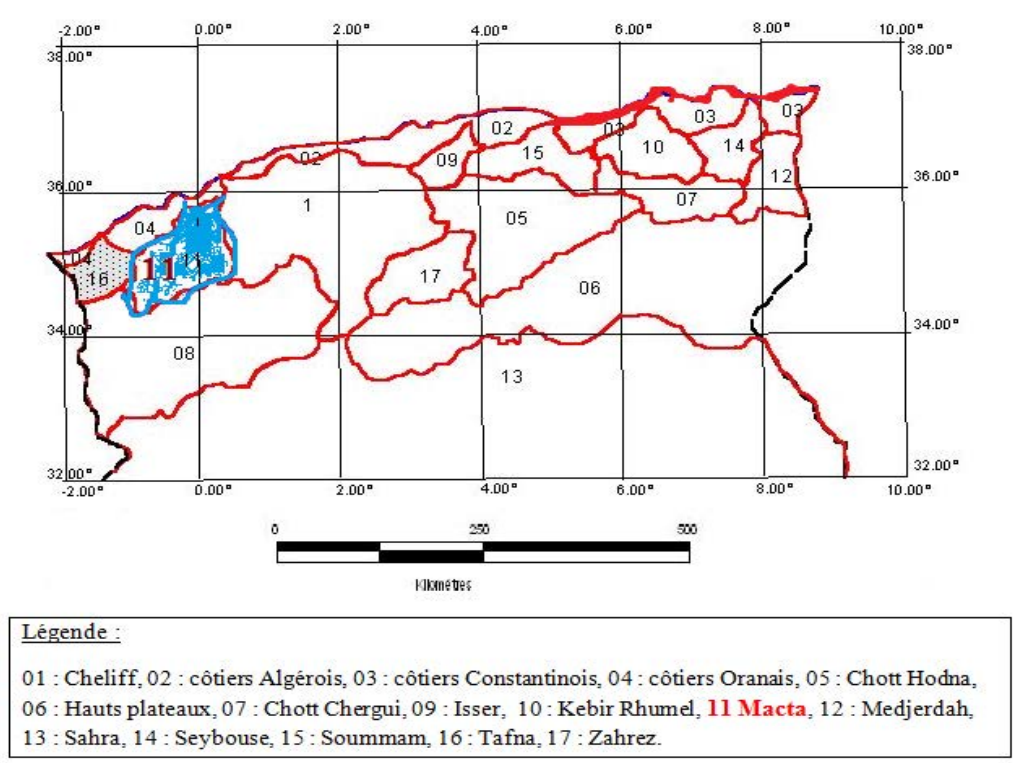

Figure 1. Bassins versants Algériens.

\subsection{Aspect climatologique}

Concernant l'aspect climatologique, en général le bassin versant de la Macta subit un climat méditerranéen au nord et un climat semi aride au sud. On recense seize postes pluviométriques qui fournissent des données remontant jusqu’à 1930. Les précipitations 


\section{XIII ${ }^{\text {èmes }}$ Journées Nationales Génie Côtier - Génie Civil \\ Dunkerque, 2-4 juillet 2014}

annuelles moyennes sur la période 1930 à 2002 varient de 230 à 659 mm (MEDDI et al., 2009).

\subsection{Couvert végétal}

Nous remarquons au niveau des confluences des affluents qui alimentent le barrage de Bouhanifia, un fort couvert végétal en Eucalyptus, les Salicacées et les chênes (voir figure 2).

Au niveau des berges des affluents, nous remarquons la présence des espèces comme les Neriums Oleder et les Arundos Donax (voir figures 3).

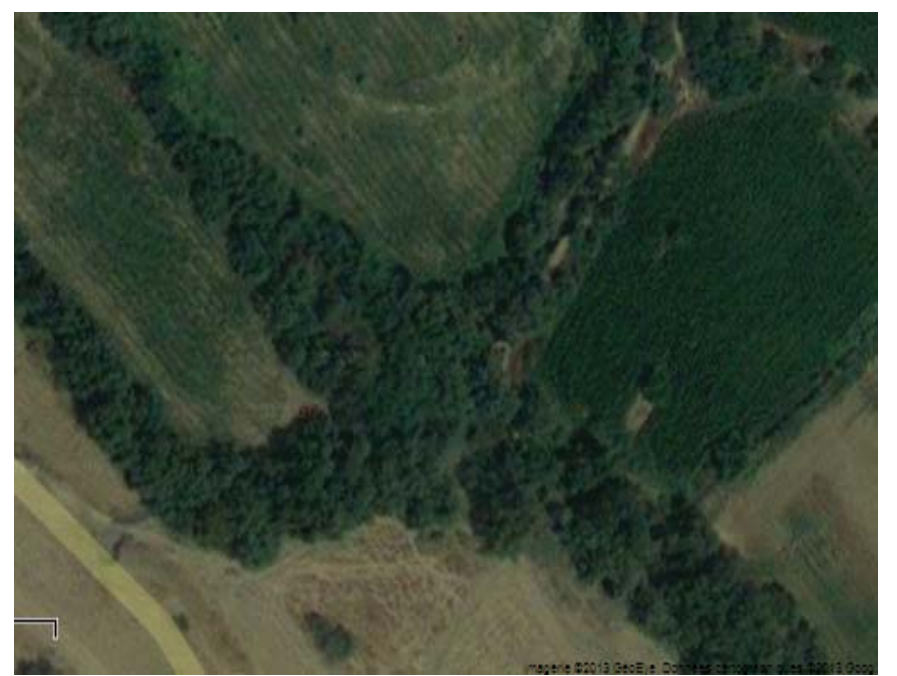

Figure 2. Couvert végétal au niveau des confluences oued Taria et Aine Fekane.
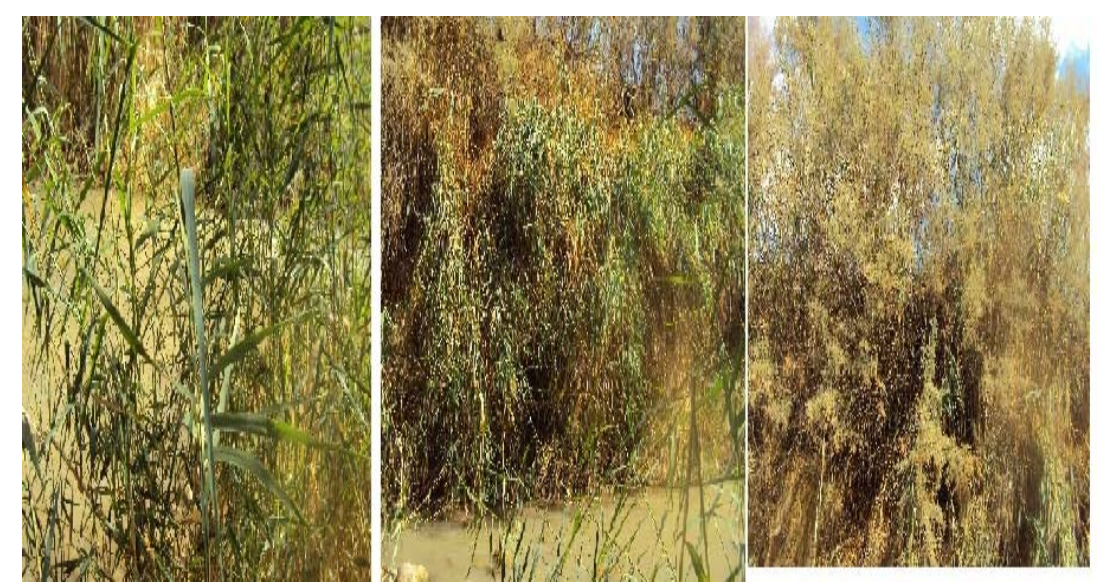

Figures 3. Espèces végétal au niveau de l'oued El Hammam.

\section{Problématique de l'envasement du barrage de Bouhanifia}

Nous avons utilisé l'équation 1 ci-dessous pour l'estimation du taux d'envasement du barrage de Bouhanifia (REMINI \& HALLOUCHE, 2005).

Tenv \% $=1,6228 t^{0,8707}$ 


\section{Thème 2 - Dynamique sédimentaire}

où : $T$ est le taux d'envasement et $t$ le nombre d'années d'exploitation.

Le tableau 1 représente l'estimation de l'évolution de l'envasement en fonction du temps. Nous pouvons remarquer que l'envasement prend de l'ampleur d'année en année, jusqu'à un comblement total en 2062. Cette prévision est susceptible de remettre en cause les méthodes de luttes actuelles.

Tableau 1. Evolution de l'envasement en fonction du temps.

\begin{tabular}{lllllll}
\hline Année & $\mathbf{1 9 4 8}$ & $\mathbf{1 9 5 5}$ & $\mathbf{1 9 7 5}$ & $\mathbf{1 9 8 6}$ & $\mathbf{2 0 1 2}$ & $\mathbf{2 0 6 2}$ \\
\hline Mesure bathymétrique de l'envasement $\mathrm{Mm}^{3}$ & 0 & 12 & 20 & 23 & $/$ & $/$ \\
Envasement calculé (1) $\mathrm{Mm}^{3}$ & 0 & 13,16 & 15,73 & 28 & 45 & 73 \\
\hline
\end{tabular}

\section{Données et méthodologie}

Dans le but de lutter contre le phénomène de l'envasement du barrage de Bouhanifia, il fallait détecter les zones productrices de sédiments et d'aborder la problématique par une approche à deux volets.

- Le premier volet est d'ordre expérimental, où neuf échantillons ont été prélevés au niveau des berges des affluents qui alimentent le barrage de Bouhanifia et un autre échantillon (à l'état vaseux) enlevé à la sortie de la vidange de fond du barrage. Cette partie d'étude permet d'identifier les sols dans les deux zones pour prévoir les solutions à adapter pour chaque type de sol (voir figures 4 et 5).

- Le second volet est de confirmer les études antérieures dont l'objectif est de distinguer la zone responsable de la mobilisation des sédiments du barrage, par une comparaison qualitative des résultats des analyses physico-chimiques des différents échantillons prélevés

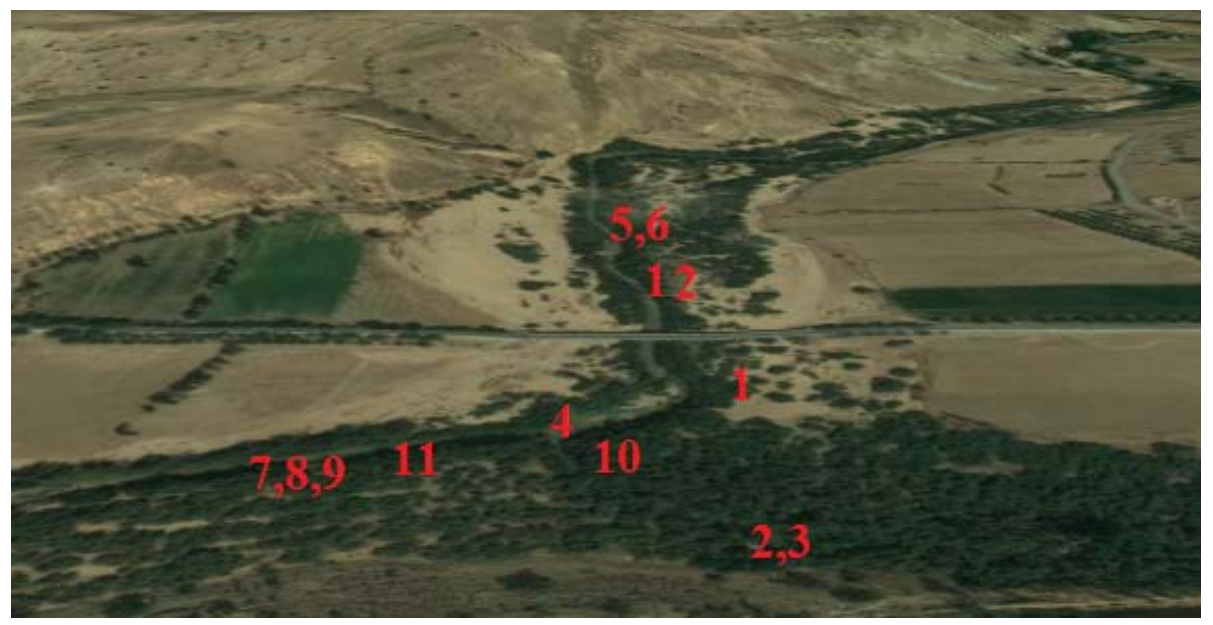

Figure 4. Vue aérienne des points de prélèvement.

\subsection{Résultats des analyses physico-chimiques des échantillons}

Les résultats des analyses physico-chimiques sont représentés dan les tableaux 2 et 3. 


\section{XIII ${ }^{\text {èmes }}$ Journées Nationales Génie Côtier - Génie Civil \\ Dunkerque, 2-4 juillet 2014}

Tableau 2. Résultats des analyses physico-chimiques.

\begin{tabular}{llllllllllll}
\hline Echantillon & $\mathbf{1}$ & $\mathbf{2}$ & $\mathbf{3}$ & $\mathbf{4}$ & $\mathbf{5}$ & $\mathbf{6}$ & $\mathbf{7}$ & $\mathbf{8}$ & $\mathbf{9}$ & $\begin{array}{c}\text { Vidange } \\
\text { de fond }\end{array}$ \\
\hline Nom de l'oued & El & Aine & Aine & Confluent & El & El & Séfouine & Séfouine & Séfouine & $/$ \\
& Hammam & Fekane & Fekane & & Hammam & Hammam & & & & \\
\hline$p H$ & 8,79 & 8,45 & 8,50 & 7,63 & 8,45 & 8,41 & 8,54 & 8,18 & 8,70 & 8,75 \\
CE ( $\mu$ s/cm) & 93,5 & 226 & 155 & 240 & 270 & 85 & 123 & 200 & 310 & 204 \\
MO (\%) & 0,56 & 0,64 & 0,21 & 0,43 & 0,30 & 0,39 & 0,47 & 1,12 & 0,43 & 0,77 \\
CaCo $(\%)$ & 10,99 & 17,75 & 8,45 & 11,73 & 21,8 & 29,94 & 15,12 & 21,97 & 14,79 & 10,99 \\
K (cm/h) & 2,35 & 0,31 & 0,49 & 0,89 & 2,61 & 2,18 & 2,73 & 0,74 & 0,59 & 3,43 \\
CEC méq/100g) & 8,30 & 17,60 & 11,33 & 25,4 & 13,2 & 15,8 & 16,55 & 14,33 & 7,5 & 8,35 \\
W (\%) & 13,5 & 19 & 18 & 15 & 15,75 & 11 & 21 & 23 & 14 & 20 \\
WL (\%) & 24 & 37 & 36 & 25 & 13 & 20 & 35 & 34 & 19 & 28 \\
Wp (\%) & 15 & 22 & 20 & 18 & 19 & 14 & 22 & 22 & 15 & 21 \\
Ip (\%) & 9 & 15 & 16 & 7 & 4 & 6 & 13 & 13 & 4 & 17 \\
Ic & 1,16 & 1,2 & 1,12 & 0,42 & 0,8 & 0,5 & 1,07 & 0,92 & 0,25 & 1,05 \\
\hline
\end{tabular}

Notations : $\mathrm{pH}$ : $\mathrm{pH}$ mètre ; $\mathrm{CE}$ : conductivité électrique ; $\mathrm{MO}$ : matières organiques ; $\mathrm{CaCO}_{3}$ : carbonate de calcium ; $K$ : coefficient de perméabilité ; CEC : capacité d'échange cationique ; $W$ : teneur en eau ; $W_{L}$ : limite de liquidité ; Wp : limite de plasticité ; Ip : indice de plasticité, Ic : indice de consistance ; A : argile ; LF : limon fin ; LG : limon grossier, SF : sable fin ; SG : sable grossier. Remarque : l'échantillon 4 est positionné par rapport au confluent des trois oueds (El Hammam, Aine Fekane et Séfioune).

Tableau 3. Résultats des analyses granulométriques.

\begin{tabular}{lllllll}
\hline Echantillon & $\boldsymbol{A}(\%)$ & LF (\%) & LG (\%) & SF (\%) & SG (\%) & Texture \\
\hline 1 & 14,88 & 3,72 & 16,74 & 31,99 & 32,66 & Sable Fin \\
2 & 20,99 & 24,49 & 33,17 & 19,94 & 1,4 & Limon Argileux \\
3 & 18,52 & 21,35 & 37,22 & 19,33 & 3,45 & Limon Argileux \\
4 & 32,88 & 8,22 & 5,89 & 44,19 & 8,88 & Limon Argileux Sableux \\
5 & 14,94 & 9,96 & 18,15 & 27,38 & 29,57 & Sable Limoneux \\
6 & 12,48 & 13,56 & 16,43 & 41,06 & 15,96 & Sable Limoneux \\
7 & 16,43 & 43,12 & 36,45 & 2,60 & 1,28 & Limon Argileux \\
8 & 18,63 & 37,99 & 33,05 & 7,45 & 2 & Limon Argileux \\
9 & 9,32 & 7,45 & 21,52 & 46,31 & 14,98 & Sable Fin \\
Vidange de fond & 16,06 & 35,34 & 43,78 & 4,43 & 0,39 & Limon Argileux \\
\hline
\end{tabular}

Après comparaison des résultats des analyses physico-chimiques des deux tableaux 2 et 3 , des échantillons prélevés aux niveaux des affluents et la vidange de fond, nous pouvons conclure que sur le plan qualitatif, les échantillons 2 et 3 prélevés à l’oued Ain Fekane, contribuent substantiellement dans la formation de la vase du barrage Bouhanifia.

\subsection{Diagnostic hydro morphologique des affluents du barrage de Bouhanifia}

L'objectif de ce diagnostic est de rendre compte du constat réel du bilan hydro morphologique des principaux affluents du barrage de Bouhanifia dans leur environnement à l'état réel. Nous pouvons par la suite, proposer des orientations de réhabilitation, de valorisation et de gestion de ces cours d'eau. 


\subsubsection{Sectorisation}

La typologie des cours d'eau n'est que la classification des tronçons de chacun (MALAVIO \& BAVARD, 2007). On arrive à la conclusion suivante que tout cours d'eau peut en théorie être segmenté en un certain nombre de tronçons. On peut dans ce cas, proposer une stratégie d'intervention par secteur homogène du cours d'eau, ou pour des sites ponctuels exposés à des risques particuliers. La figure 5 représente la sectorisation des cours d'eau du bassin versant de Bouhanifia.

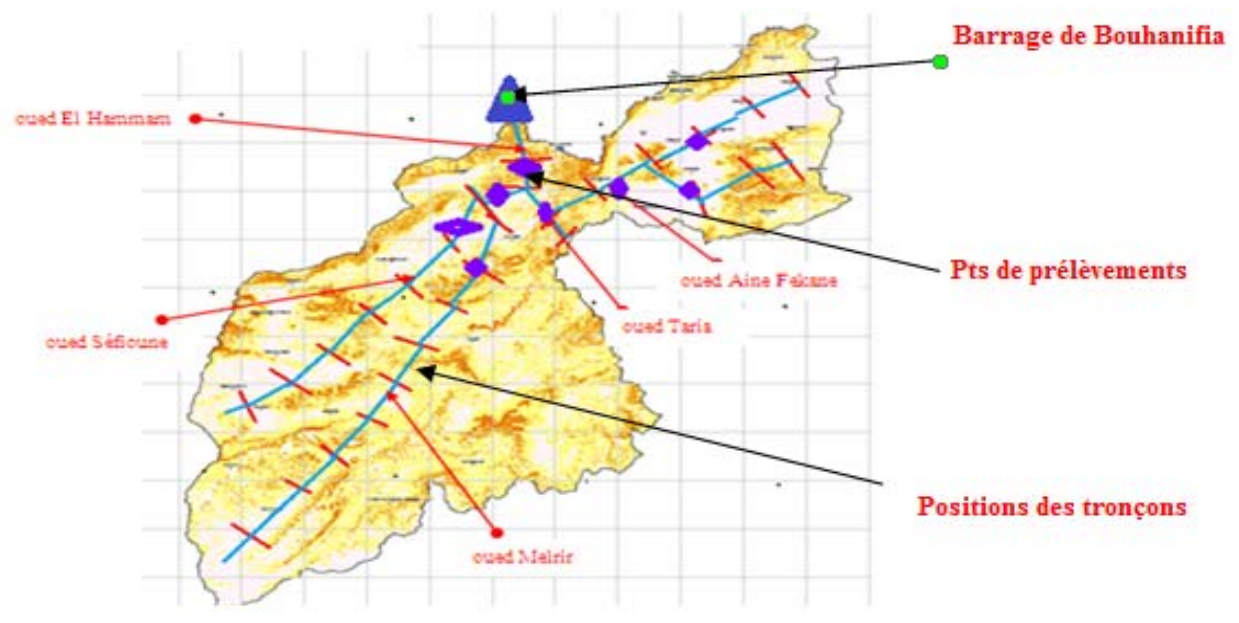

Figure 5. Sectorisation des cours d'eau du bassin versant du barrage de Bouhanifia.

\subsubsection{Les pentes}

A partir d'un MNT (Modèle Numérique des Terrains) donnant la zone d'étude et après un traitement par le logiciel Global Mapper, nous avons pu déterminer les pentes de chaque oued, voir tableau 4.

Tableau 4. Pentes des tronçons des oueds Melrir, Séfioune, Aine Fekane et Taria.

\begin{tabular}{lllll}
\hline $\boldsymbol{N}^{\circ}$ du tronçon & $\begin{array}{l}\text { Oued Merir } \\
\text { Pente \%o }\end{array}$ & $\begin{array}{l}\text { Oued Séfouine } \\
\text { Pente \%o }\end{array}$ & $\begin{array}{l}\text { Oued Ain Fekane } \\
\text { Pente \%o }\end{array}$ & $\begin{array}{l}\text { Oued Taria } \\
\text { Pente \%o }\end{array}$ \\
\hline 1 & 3 & 2,5 & 5 & 7 \\
2 & 2,5 & 1,5 & 3 & 5 \\
3 & 2,5 & 0,5 & 2,5 & 2,5 \\
4 & 2 & 0,75 & 1,75 & 1,5 \\
5 & 0,5 & 1,5 & $/$ & 1,5 \\
6 & 0,2 & 0,5 & $/$ & 0,25 \\
7 & 0,2 & $/$ & $/$ & $/$ \\
8 & 0,12 & $/$ & $/$ & $/$ \\
\hline
\end{tabular}




\section{XIII ${ }^{\text {èmes }}$ Journées Nationales Génie Côtier - Génie Civil \\ Dunkerque, 2-4 juillet 2014}

La figure 6 permet de visualiser la variation de chaque cours d'eau considéré en fonction des différentes pentes de chacun.

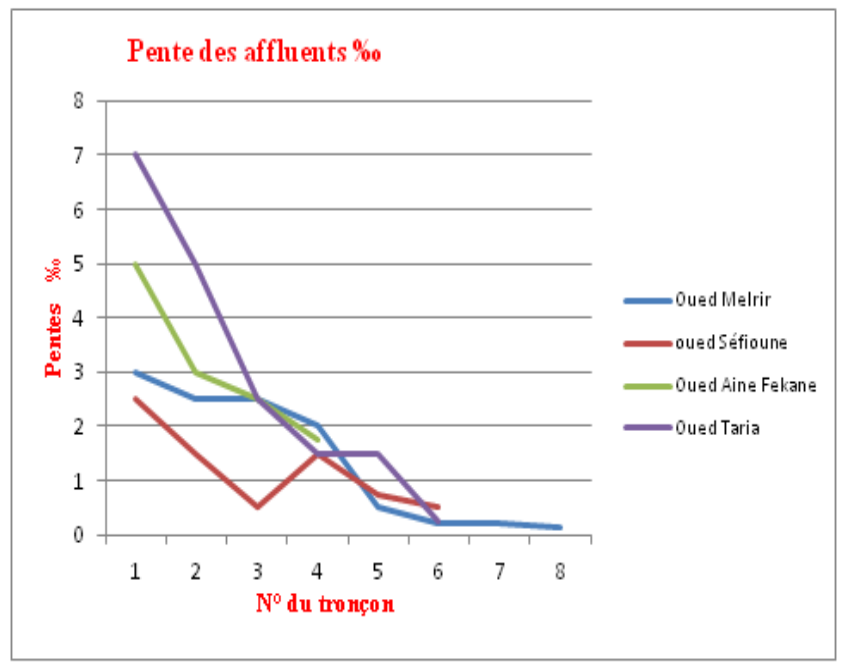

Figure 6. Les pentes des tronçons des affluents du barrage de Bouhanifia.

\subsubsection{La sinuosité}

La sinuosité est un paramètre principal du lit d'un cours d'eau qui est défini par le coefficient $r$. Ce coefficient est le rapport de la longueur développée du cours d'eau avec la longueur la plus courte au fond de sa vallée. En ce qui concerne les sinuosités, nous avons utilisé le calcul direct sur les cartes, voir tableau 5. Les précisions de calcul des pentes et des sinuosités sont approximatives, car même in situ la détermination des pentes ne pourrait être précise.

Tableau.5. Coefficient de sinuosité r des tronçons des oueds Melrir, Séfioune, Aine Fekane et Taria.

\begin{tabular}{lllll}
\hline $\boldsymbol{N}^{\circ}$ du tronçon & $\begin{array}{l}\text { Oued Merir } \\
(\boldsymbol{r})\end{array}$ & $\begin{array}{l}\text { Oued Séfouine } \\
(\boldsymbol{r})\end{array}$ & $\begin{array}{l}\text { Oued Ain Fekane } \\
(\boldsymbol{r})\end{array}$ & $\begin{array}{l}\text { Oued Taria } \\
(\boldsymbol{r})\end{array}$ \\
\hline 1 & $/$ & $/$ & 2,04 & 3,34 \\
2 & $/$ & $/$ & 2 & 2,5 \\
3 & $/$ & $/$ & 1,04 & 1,25 \\
4 & $/$ & $/$ & 1,15 & 4,6 \\
5 & $/$ & $/$ & $/$ & 1,5 \\
6 & 1,5 & 2 & $/$ & 3,54 \\
7 & 2,03 & 3,2 & $/$ & 3,05 \\
8 & 3,4 & & & 1,25 \\
\hline
\end{tabular}

Nous montrons sur la figure 7 , la variation du coefficient de sinuosité en fonction des tronçons. 


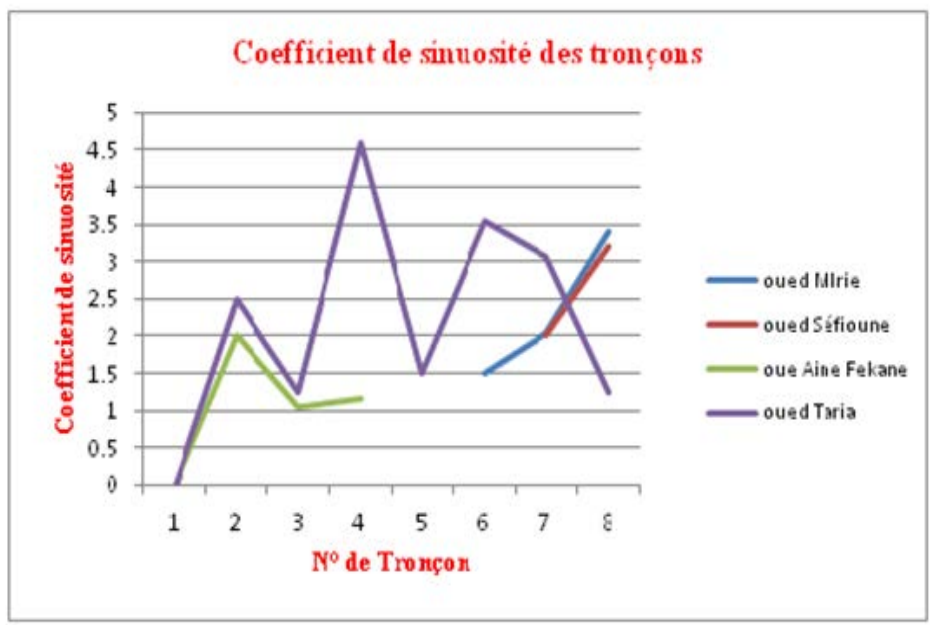

Figure 7. Les Sinuosités des tronçons des affluents du barrage de Bouhanifia.

\subsubsection{La rugosité}

Trois autres échantillons (10, 11 et 12) ont été prélevés en profondeur au niveau de la confluence des oueds Aine Fekane et Taria (échantillon 10), et ceux d'El Hammam (échantillon 11) et Sefioune (échantillon 12), voir figures 4 et 5 . Les courbes granulométriques des trois échantillons (voir figure 8) montrent que les valeurs de $d_{50}$ sont de 2,$5 ; 3,5$ et $3 \mathrm{~mm}$.

La granulométrie des alluvions est presque homogène et supérieur à 2,5, d'où une rugosité importante des lits des affluents. Cette particularité participe à la dissipation de l'énergie cinétique de l'eau.

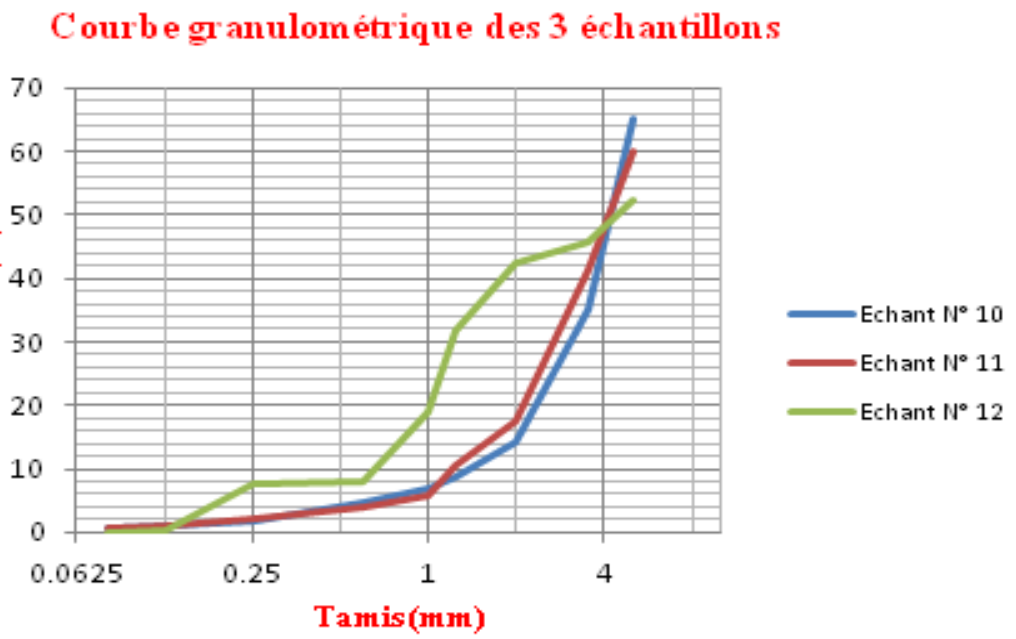

Figure 8. Courbes granulométriques. 


\section{XIII ${ }^{\text {èmes }}$ Journées Nationales Génie Côtier - Génie Civil \\ Dunkerque, 2-4 juillet 2014}

Après le diagnostic de l'état physique et du fonctionnement hydro morphologique des affluents qui alimentent le barrage de Bouhanifia, nous avons constaté ce qui suit (voir figure 5) :

- Les oueds Melrir et Séfioune ont tendance à s'auto-équilibrer, à cause de leurs pentes moyennes $(0,5 \%$ à $3 \%$ ) et de leur faible sinuosité, ce qui minimise leur contribution dans l'envasement du barrage Bouhanifia.

- L'oued Taria est du type méandre, à cause de sa forte sinuosité et ses pentes assez variées, ainsi que la nature du sol favorisant l'érosion de ses berges. L'oued Ain Fekane est le prolongement naturel à l'aval de l'oued Taria, malgré son profil en long plutôt rectiligne, il subit les conséquences de l'oued Taria du point de vue apport solide.

Donc l'oued Taria et son prolongement, ainsi que l'oued Ain Fekane sont en déséquilibre morpho-sédimentaire, ce qui les rend potentiellement responsables de l'envasement du barrage de Bouhanifia. De ce fait, cette zone nécessite des interventions de maintenance et d'entretien, pour minimiser l'érosion et par conséquent contribuer à l'entretien du barrage de Bouhanifia en aval contre l'envasement.

\section{Maintenance préventive du barrage de Bouhanifia}

Le volume important des sédiments qui est transporté de l'amont du bassin versant vers la retenue du barrage de Bouhanifia, est le plus grand problème qui influence sur la capacité de la retenue, la sécurité de l'ouvrage, sa durée de vie et de son environnement. Pour luter contre ce phénomène, il existe plusieurs méthodes qu'ont peut les classer sur deux types de mesures, à savoir des mesures curatives et des mesures préventives.

\subsection{Mesures curatives}

- Construction de barrages de décantation.

- Surélévation des barrages.

- Les chasses dites à l'espagnole.

- Evacuation des courants de densité.

- Dragages des retenues.

\subsection{Les insuffisances des mesures curatives}

D’après CHADI et HADJI (1992) ; on constate ce qui suit :

- L'inconvénient du barrage de décantation ou appelé nouveau barrage, est qu'il n'est pas toujours facile de trouver des conditions favorables répondants aux critères de réalisation.

- Les surélévations des barrages en Algérie ne peuvent être réalisées que pour ceux qui sont en béton.

- Pour le dragage des barrages, le problème majeur est le rejet de la vase dans des zones de stockage respectant l'environnement. Malgré les études faites concernant la 


\section{Thème 2 - Dynamique sédimentaire}

valorisation de la vase, un deuxième problème majeur est celui des coûts très élevés de l’opération du dragage ainsi que la quantité d'eau élevée perdue.

- Les chasses et l'utilisation des courants de densité consomment des quantités importantes d'eau souvent $10 \mathrm{~m}^{3}$ pour $1 \mathrm{~m}^{3}$ de vase évacué.

Pour palier ses insuffisances, les mesures préventives restent sans doute le moyen le plus efficace.

\subsection{Mesures préventives}

Les mesures préventives sont toutes des actions qui servent à minimiser l'érosion en amont du barrage, on peut citer les dispositifs suivants (voir figure 9).

\subsection{Les revêtements des berges}

Les revêtements des berges sont des ouvrages destinés à protéger une rive menacée d'érosion, ou reconstituer un talus de berge érodé et aussi à remettre en état un ouvrage de protection défaillant. Il existe plusieurs types de revêtement des berges qui dépendent tous de la zone ou de l'étage du talus. On distingue trois étages successifs : (voir figure 9A).

a) La zone située au-dessous du niveau des basses eaux et dont l'immersion est permanente. Dans cette zone, le placage d'enrochement est la règle générale, sauf en cas de la présence de sable boulant ou argile fluente, on les renforce par des rideaux métalliques

b) La zone située au-dessus du niveau des crues quinquennales, l'émersion est quasi permanente ; c’est le tapis végétal qui est généralement proposé comme solution.

c) La zone intermédiaire avec des revêtements constitués par un placage d'enrochement ou moellons de gros éléments, composé aussi d'éléments plus petits destinés à réduire les vides interstitiels.

\subsection{Les épis}

Les épis constituent une méthode indirecte permettant de limiter l'érosion, ils consistent à dévier les vitesses d'écoulement élevées pour les éloigner des berges érodables. Ces structures fonctionnent d'une façon autonome en assurant plusieurs fonctions (voir figures 9B et 9C) :

- stabiliser le bras du cours d'eau pour maintenir le chenal à faible hauteur d'eau dans une position favorable ;

- resserrer le chenal à faible hauteur d'eau pour augmenter sa profondeur ;

- protéger les berges en éloignant l'écoulement principal, on remarque que les vitesses du courant près des berges sont généralement réduites de moins de $50 \%$ de leurs valeurs initiales. 


\section{XIII ${ }^{\text {èmes }}$ Journées Nationales Génie Côtier - Génie Civil \\ Dunkerque, 2-4 juillet 2014}

\subsection{Digues longitudinales}

Les digues longitudinales (voir figure 9D) ont deux fonctions, à savoir :

- stabiliser le canal,

- guider l'écoulement.

En effet, d'une part les digues longitudinales permettent de prévenir l'érosion en périodes de débit fort, et facilitent le dépôt des sédiments entre la digue et la berge en période de faible hauteur. D'autre part, elles permettent d'empêcher tout débordement en période de crue et de guider l'écoulement, car les digues sont conçues avec des crêtes suffisamment hautes.

\subsection{Génie végétal}

Le génie végétal est l'ensemble des techniques rapprochant les principes de l'écologie et du génie. La stabilisation des talus de berges et des rives est conçue de telle façon à permettre d'avoir des ouvrages qui utilisent des végétaux comme matériel de base (HELENE, 2010), (voir figures 9E et 9F).

En ce qui concerne le cas de l'oued Taria, on remarque une forte succession des méandres qui prennent différents formes sinueuses. Nous proposons les solutions suivantes (voir figure 5 ) :

- Pour les tronçons 3, 5 et 8: une combinaison de deux techniques. La fascine d'hélophytes qui est réalisée avec des végétaux semi-aquatiques plantés dans un boudin de géotextile rempli de matériaux terreux et fixé par une ou deux rangés de pieux (voir figure 9E) et un revêtement des berges en maçonneries (voir figure 9A);

- Pour les tronçons 1, 2, 4, 6 et 7: des épis en bétons armés inclinés et orientés dans le sens contraire de l'écoulement (voir figures 9B et 9C).

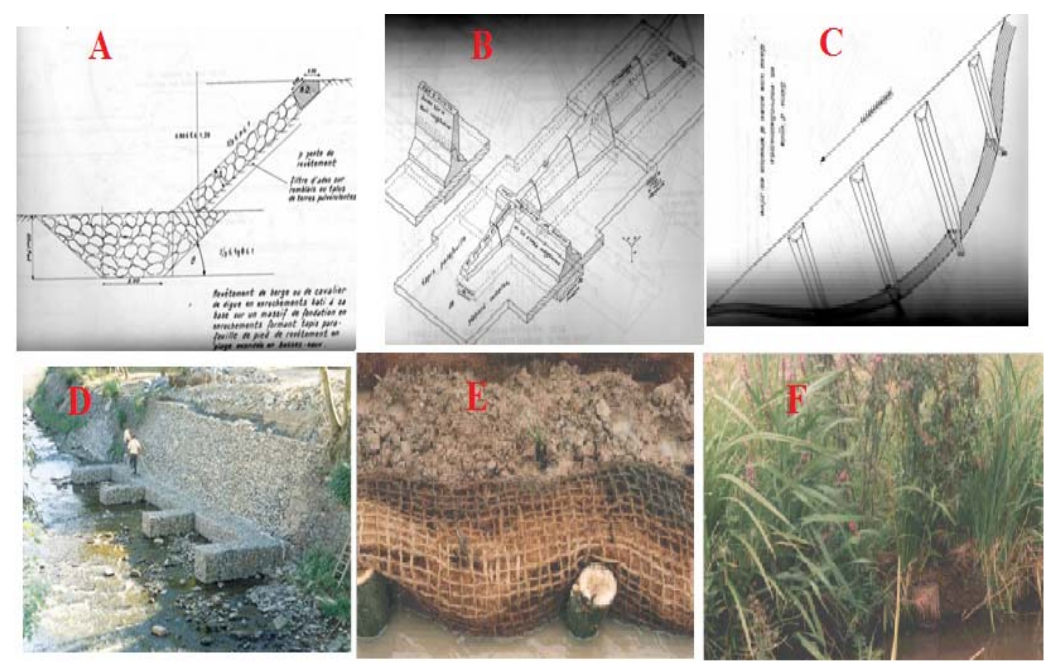

Figures 9. Dispositifs des mesures préventives contre l'érosion. 


\section{Conclusions}

$\mathrm{Au}$ vu des résultats des essais d'identification des sols qui forment les berges des affluents alimentant le barrage de Bouhanifia, et après diagnostic de leurs états de fonctionnement morpho-sédimentaire, nous avons pu localiser la zone productrice de sédiments. La zone est du bassin versant est celle dont les sédiments atterrissent au niveau des oueds Aine Fekane et Taria.

Afin de protéger les berges contre l'érosion, nous avons proposé des techniques qui ont prouvé leur efficacité dans la région méditerranéenne, notamment du coté sud de la région d'étude (région semi aride).

\section{Références bibliographiques}

BOUANANI A. (1999). Hydrologie, Transport Solide et modélisation étude de quelques sous bassins de la Tafna (Nord Ouest de l'Algérie). Thèse de Doctorat d'Etat de l’Université Abou Bekr Belkaid de Tlemcen., 249 p.

CHADI A., HADJI T. (1992). Erosion des sols et envasement des barrages. Algérie Equipement., Revue Technique de l'Ecole Nationale des Travaux Publiques, №5, pp 20-25.

GOMER D. (1994). Ecoulement et érosion dans les petits bassins versants à sol marneux sous climat semi-aride méditerranéen. Traduction française (Paschen $\mathrm{H}$. et Vogt H.) thèse de l'université technique de Karlsruhe. Agence Nationale Ressources Hydrauliques (Algérie) et Deutsche Gesellschaft für Technische Zuzammenarbeit Gmbh (Allemagne). Projet Pilote d'Aménagement Intégré du BV de l'Oued Mina, 207 p.

HELENE M. (2010). La restauration des berges. Fiche technique, diagnostic et solution des problèmes d'érosion des cours d'eau. Mémoire maître en environnement, Université de Sherbrooke, 99 p.

MALAVIO J.R., BAVARD J.P. (2007). Elément d'hydro morphologie fluvial. Office nationale de l'eau et des milieux aquatiques (ONEMA). Collection Armand Colin, Masson, $224 \mathrm{p}$.

MEDDI M., TALIA A., MARTIN C. (2009). Evolution récente des conditions climatiques et des écoulements sur le bassin versant de La Macta nord ouest de l'Algérie. Article scientifique, Géographie physique et environnement, Vol. III, pp 61-84.

REMINI B., HALLOUCHE W. (2005). Prévision de l'envasement dans les barrages du Maghreb. Larhyss journal, ${ }^{\circ}$ 4, juin 2005, pp 69-80. 